1 Phenotypic selection on nectar amino acid composition in the Lepidoptera

2

3

4

5

$6{ }^{1}$ Plant Conservation and Population Biology, Biology Department, University of Leuven,

$7 \quad$ Kasteelpark Arenberg 31, B-3001 Heverlee, Belgium.

$8 \quad{ }^{2}$ Lab of Molecular Plant Biology, Biology Department, University of Leuven, Kasteelpark

9 Arenberg 31, B-3001 Heverlee, Belgium.

10

11 * Correspondence author.

12 E-mail: Pieter.Gijbels @ bio.kuleuven.be

13

Fax: 003216321968 
Plant-pollinator interactions are potential drivers of evolution in floral traits. Because nectar chemical composition is known to mediate both plant-pollinator interactions and plant reproductive success, it can be expected that chemical composition of nectar is subjected to strong pollinator-mediated selective forces. However, the extent of natural selection on different nectar components has not been studied so far. Using the Lepidoptera pollinated fragrant orchid Gymnadenia conopsea as a model species, we used high-performance anionexchange chromatography (HPAEC) to characterize the sugar and amino acid composition of floral nectar in three calcareous grassland populations of $G$. conopsea. We then measured phenotypic selection on nectar composition and on other plant and floral traits through applying both linear regression and structural equation modelling. We demonstrate phenotypic selection on plant height, inflorescence height and on specific nectar amino acids, whereas spur length, total sugar and amino acid concentration were not direct targets of selection. Chemical nectar composition is thus indeed under selective pressure but nectar amino acids are much more important to fitness of G. conopsea, as compared to nectar sugars. Furthermore, as we found no evidence of selection on the total amino acid concentration, it is unlikely that amino acids increase pollinator attraction because they are a pollinator nitrogen source. To further unravel the evolutionary ecology of floral nectar, behavioural experiments with pollinators exposed to different nectar components and studies experimentally identifying the selective agents are recommended. 
Plant-pollinator interactions are expected to drive floral evolution (Fenster et al. 2004), and the prevalence of pollen limited female reproductive success suggests ample potential for pollinator-mediated selection on flower characteristics (Knight et al. 2005). These predictions have been confirmed by numerous studies reporting phenotypic selection on various floral traits (reviewed in Harder and Johnson 2009). Selection has been demonstrated for traits that mediate either pollinator attraction (e.g. plant height, number of flowers and flower size (Mitchell et al. 2004, Armbruster et al. 2005)) or pollinator efficiency (e.g. corolla tube width, corolla tube length and spur length (Campbell et al. 1996, Bloch and Erhardt 2008, Sletvold et al. 2010)).

Next to flower morphology and flower display, nectar chemical composition is also known to mediate plant-pollinator interactions (Petanidou 2005, González-Teuber and Heil 2009, Heil 2011, Nepi 2014), and to affect pollinator attraction and plant reproductive success (Leiss and Klinkhamer 2005, Gijbels et al. 2014). Nectar is a sweet and chemically complex solution, with sugars and amino acids (AAs) as the most important constituents (Nicolson and Thornburg 2007). Both concentration and composition of nectar sugars have been shown to correlate with specific responses of pollinators and with a plant species' pollination syndrome (Baker and Baker 1990, Wolff 2006). Hummingbirds, Lepidoptera and long-tongued bees, for example, usually prefer nectars rich in the disaccharide sucrose (Suc), whereas short-tongued bees and flies prefer nectar rich in the hexose monosaccharides glucose (Glc) and fructose (Fru) (Baker and Baker 1983, Petanidou 2005, Krömer et al. 2008). Similarly, nectar AA profiles were found to be related with specific pollination syndromes (Baker and Baker 1986). For example, high nectar AA concentrations have been reported in plants pollinated by Lepidoptera and by other pollinators which cannot feed on pollen, and hence are dependent on nectar AA as a nitrogen source (Baker and Baker 1986, Gijbels et al. 2014). Insect pollinators have also been shown to exhibit specific behavioural responses to AAs. Butterflies and bees, for example, have been experimentally demonstrated to be able to detect single AAs in nectar sugar solutions and to prefer nectar mimics containing specific mixtures of AAs (Alm et al. 1990). All these findings have led to the general expectation that nectar composition is subjected to strong pollinator-mediated selective forces capable of rapidly changing the chemical composition of nectar (Baker and Baker 1990, Leiss and Klinkhamer 2005, Jacquemyn et al. 2012).

Recently, evidence has accumulated, however, that both nectar sugar and AA composition are highly variable, not only among populations and among individuals within populations (Farkas et al. 2012, Nocentini et al. 2013), but also among flowers within a plant 
73 individual (Herrera et al. 2006, Gijbels et al. 2014). Yet, phenotypic selection on nectar traits can only occur at the level of an individual, and even if pollinators prefer a specific type of nectar, their selective potential on floral nectar is very likely to be strongly hampered by the variation in nectar composition among flowers within individuals. However, whether or not phenotypic selection on nectar chemical composition occurs in natural populations has not been investigated. This restriction in our understanding of the potential for evolution of nectar chemical composition was the main motivation for this study.

We used high performance anion exchange chromatography with pulsed amperometric detection (HPAEC-PAD) to analyze nectar sugar and AA composition in three populations of the Lepidoptera pollinated orchid Gymnadenia conopsea. Former work on this species has shown high within individual variation in chemical nectar traits, but also significant correlations between fruiting success and specific nectar AA profiles (Gijbels et al. 2014). We applied two complementary methodologies with the aim of detecting the presence and the extent of phenotypic selection on nectar composition.. 


\section{Species and study sites}

G. conopsea (the Fragrant orchid) is a perennial, terrestrial orchid, widely distributed throughout Eurasia where the species grows in open meadows from the lowlands up to subalpine levels (Komarov 1986). G. conopsea flowers in the middle of summer and produces one inflorescence which consists of a dense spike of 10-80 pale pink to intense violet flowers (Meekers et al. 2012). The flowers produce a scent composed of 45 different volatiles including the physiologically active benzyl acetate, eugenol and benzyl benzoate (Huber et al. 2004). Flowers have a long spur half-filled with Suc dominant nectar, rich in AAs (Gijbels et al. 2014). G. conopsea is self-compatible, but since spontaneous autogamy or apomixis does not occur, successful fruit set is still pollinator dependent (Gustafsson 2000). Floral traits of G. conopsea indicate generalization towards a wide range of long-tongued Lepidoptera species and pollinators include different species of diurnal and nocturnal moths and butterflies (Gustafsson 2000, Huber et al. 2004, Sletvold et al. 2012). Earlier studies on this species have already shown that there is positive pollinator mediated selection on floral display and spur length (Sletvold and Ågren 2010). The three populations we studied grow on dry calcareous grasslands at three different localities (Coupu Tienne, Les Hurees and Gay) in the valley of the river Viroin in the south-western part of the Calestienne region, Belgium (see Adriaens et al. 2006) for a full description of the study area). Due to the highly fragmented nature of the studied grasslands, regular gene flow between populations is highly unlikely. Population sizes ranged from 56 to 541 flowering individuals, and populations were located within $6 \mathrm{~km}$ of each other (Table 1).

\section{Field survey and nectar analyses}

At the onset of flowering (June 15th 2011), 50 individuals were selected in the 2 largest populations, and 35 in the smallest populations. Plant heights (distance from the ground to the topmost flower) and inflorescence height (distance from the lowermost flower to the topmost flower) were recorded for all selected individuals, and the height of the surrounding vegetation was measured. Inflorescence height strongly correlates with number of flowers in an inflorescence of $G$. conopsea (Personal data: $\mathrm{n}=100$, Pearson correlation $=0.71, \mathrm{P}<0.001$ ) and is therefore a good indicator of floral display. We collected two of the lowermost flowers from each individual and measured spur length (distance from corolla to spur tip) to the nearest 0.01 $\mathrm{mm}$, using digital photography and image analyses software (Image J, Maryland, USA) (supplementary material Appendix 1, Fig. A1). 
Nectar samples of all populations were collected during two consecutive mornings just before the flowering peak. The day prior to nectar collection, the inflorescence of selected individuals was bagged with a $1 \mathrm{~mm}$-mesh to allow nectar build up in the spurs. The following morning, three to four of the most recently opened flowers per inflorescence were selected for nectar collection. Nectar was retrieved from the spur using $5 \mu \mathrm{l}$ microcapillary tubes (Hirschmann Laborgeräte $\mathrm{GmbH} \& \mathrm{Co} . \mathrm{KG}$, Eberstadt, Germany) and cooled $\left(4{ }^{\circ} \mathrm{C}\right)$ for transportation to the laboratory. Samples were then diluted with $10 \mu$ l sodium azide-water $(0.02 \% \mathrm{w} / \mathrm{v})$ and frozen $\left(-20{ }^{\circ} \mathrm{C}\right)$ until HPAEC-PAD analysis. In total we collected 453 nectar samples. Nectar composition was analyzed with a HPAEC-PAD on an ICS3000 chromatography system (Dionex, Sunnyvale, CA) as described before (Gijbels et al. 2014). Samples of different populations were analysed in a random order, i.e. individuals of a population were examined over the entire period of analysis. Nectar components detected sporadically and in low quantity were excluded from further analyses. Nectar values of individual plants are simple averages over all sampled flowers in an individual.

On August 9, 2011, we quantified female reproductive success of all sampled individuals by recording the number of fruits at maturation and the fruit mass of two nondehisced fruit capsules. The inflorescence of two individuals in Les Hurées and one in Gay could not be retrieved due to herbivory. Fruit mass is known to be positively correlated with number of seeds with embryos in G. conopsea (Sletvold and Ågren 2010). For each individual, we estimated female fitness as the product of the number of fruits and the mean fruit mass (Sletvold et al. 2010). Average plant traits per population are reported in Table 1.

The female reproductive success of plants can be restricted by both resource availability and pollen limitation (Knight et al. 2005). (Harder \& Johnson, 2009). However, Gijbels et al. (2014) showed that compared to pollen limitation, resource availability likely has a negligible influence in the study system as average fruiting success was approximately $80 \%$ in all three studied populations, and supplemental hand pollinations significantly increased fruit set to nearly a $100 \%$.

\section{Statistical analyses}

The effects of population on plant traits and plant performance were examined with a one-way ANOVA. Next relative female fitness (individual fitness divided by mean fitness) and standardized trait values of the traits plant height, spur length, total sugar concentration, Suc concentration, total AA concentration, and the concentration of all individual AAs (with a mean of 0 and a variance of 1) were calculated separately for each population. We then used 
multivariate models to estimate direct selection gradients $(\beta)$ for the pooled data across the three populations (Lande and Arnold 1983). Because nectar AA compounds were strongly correlated (Supplementary Table 1), we first conducted a Principal Component Analysis (PCA) with varimax rotation on the concentration of all different AAs and total AA concentration. Principal components (PCs) were then used as independent variables in the selection analysis, together with the standardized values of plant height, spur length, total sugar and Suc concentration. Multicollinearity was not an issue as variation inflation factors never exceeded 3 (Quinn 2002). We initially included quadratic (gii) and cross-product (gij) terms in the regression models to quantify nonlinear and correlational selection (Sletvold et al. 2010). However, none of these terms were statistically significant and they were omitted from further analysis and discussion. We also evaluated differential selection among the three studied populations using ANCOVA. The model included relative female fitness as the dependent variable and the eight standardized traits (plant height, inflorescence size, spur length, total sugar concentration, Suc concentration and the three PCs), population and the trait-population interaction as independent variables.

Through using PCA on the AA data, the problem of multicollinearity between independent variables was avoided, but simultaneously, information was lost on the potential specific relation between individual AAs and female fitness. Structural equation modelling offers a solution to this problem through accounting for any correlations between variables (Scheiner et al. 2000). Because there were too many floral traits to be included in a single path analysis (no model convergence), we only included variables that strongly correlated with female fitness (Pearson correlation with $\mathrm{P}<0.01$ ) (supplementary material Appendix 1, Table A1). Model fit to the data was tested using the likelihood chi-squared value, which tests the null hypothesis that the covariance matrix implied by the model reproduces the observed covariance matrix. Bentler's comparative fit index (CFI) was calculated to estimate goodness of fit, with values greater than 0.9 indicating an acceptable fit between the model and the data (Grace et al. 2007). Once the model was constructed, we performed backward selection by excluding the least significant variables until model fit no longer improved. Maximum likelihood estimation was used to determine the standardised path coefficients; these are equivalent to standardized selection gradients ( $\beta$ ) (Scheiner et al. 2000). 
Most plant and floral traits differed significantly between populations (Table 1) and were positively correlated with each other, especially the different AAs showed very strong intercorrelations (supplementary material Appendix 1, Table A1). The PCA produced three PCs with an eigenvalue greater than 1, together these PCs explained $79.6 \%$ of the total variance (Table 2). Using multivariate regression, we found significant positive phenotypic selection on inflorescence height and on AAs represented by PC2 (Table 3, Fig. 1). PC2 is positively correlated with the concentration of nectar AAs alanine, serine, glutamine, asparagine and threonine among others (Table 2). We found no phenotypic selection on AAs represented by PC1 (glutamic acid and arginine among others), and PC3 (total AA, glycine), on spur length, sugar or Suc concentration ( $\mathrm{P}>0.05$, Table 3 ). Phenotypic selection gradients were not different among the three studied populations (supplementary material Appendix 1, Table A2). Additional scatterplots between individual AAs or other floral traits and female reproductive success can be found in the supplementary material Appendix 1, Figure A

Spur length, sugar and Suc concentration and some AAs did not strongly correlate with female fitness $(\mathrm{P}>0.01)$, and where excluded from the structural equation models (supplementary material Appendix 1, Table A1). The full models included plant height, inflorescence height, total AA, alanine, serine, glycine, threonine, isoleucine, lycine, asparagine and histidine. The saturated structural equation model did not appropriately describe the data (the model did not fit the data, $\mathrm{P}<0.05$ ). Removing the least significant variables threonine, lysine and isoleucine improved the model. Additional omissions of non-significant variables did not further improve the model fit (Table 4). The final model demonstrated a positive phenotypic selection on plant height and inflorescence height. Similar to the multiple regression analyses, total AA concentration did not affect female fitness, but there was positive phenotypic selection on Serine (Ser), and negative phenotypic selection on alanine (Ala) (Fig. 2). 
Although chemical analytical methodology underwent great progress in the recent decade, which allowed for easier and more precise detection of nectar composition, the biological and evolutionary interpretation of variability in nectar composition is lagging behind (Brandenburg et al. 2009). The complex chemical composition of nectar is chiefly responsible, as there are a multitude of nectar variables to consider while likely not all of them have biological relevance. This study is the first to demonstrate phenotypic selection on nectar traits as we found significant selection on specific AAs, whereas selection on total sugar and AA concentration was absent.

With the exception of spur length, total AA concentration and some individual nectar AAs, all floral traits significantly differed among the three studied populations of $G$. conopsea (Table 1). It is known that G. conopsea shows large variation in both floral morphology (Sletvold et al. 2010, Meekers et al. 2012) and nectar composition (Gijbels et al. 2014). Phenotypic selection, however, did not vary among populations and hence did not contribute to the between population variation through local adaptation in floral traits (supplementary material Appendix 1, Table A2). This is in accordance with the very similar soil and vegetation conditions and landscape context of the three populations, which suggest very similar pollinator communities.

Both adopted statistical approaches demonstrated positive directional selection on inflorescence height. The effect of plant height was insignificant in the linear regression model $(\mathrm{P}=0.11$, Table 3$)$, but plant height significantly related to reproductive success in the structural equation model (Fig. 2). Both these traits contribute to floral display which has previously been shown to increase pollinator attraction and to improve plant fitness in both G. conopsea (Sletvold and Ågren 2010), and in many other animal-pollinated plant species (e.g. Mitchell et al. 2004). The strength of selection on plant height is known to vary with the local vegetation characteristics as there is stronger selection on taller plants in tall vegetation (Sletvold et al. 2013). In the studied grasslands, vegetation heights were similar (on average $14 \mathrm{~cm}$ ), and similar selection strength on taller, more attractive plants could be expected. Sletvold et al. (2010) also found directional selection on longer spurs in G. conopsea. Short spurs are expected to decrease pollinator efficiency as pollinaria from long-spurred plants sticking to the proboscis of pollinators fail to contact the stigma of short-spurred plants and hence cause stronger pollen limitation (Johnson and Steiner 1997). We found no evidence for selection on spur length, however. The abundant presence and occasional visits of Lepidoptera with a short proboscis 
such as Melanargia galathea (proboscis length is $12.7 \mathrm{~mm}$ (Bloch and Erhardt 2008)) in the study area may relax the selective pressure on spur length and contribute to the absence of a selection gradient as plants with a short spur can also receive pollen.

We did not find selection gradients for nectar sugar composition (Table 3), even though many studies have shown that Lepidoptera prefer Suc-dominated nectars over hexosedominated nectars (Rusterholz and Erhardt 1997 and references therein). However, it has also been demonstrated that up to $70 \%$ of total variation in sugar composition is situated between flowers of the same individual (Herrera et al. 2006, Gijbels et al. 2014). This high variation within individuals strongly hampers the selective potential of pollinators on nectar sugar composition. We also found no selection on total sugar concentration in G. conopsea. It is possible that sugars are not the limiting nutrients for pollinators. Plant communities growing on nutrient limited soils such as the calcareous grasslands studied create an effective excess of carbon which makes an abundant production of carbon-based floral rewards relatively cheap (Sinclair 2012). Furthermore, nectar sugars not only supply energy, they also affect the viscosity of nectar, and by extent the rate at which Lepidoptera can consume nectar (Pivnick and McNeil 1985). Therefore, nectar sugar concentration corresponds to optimal rates of Suc uptake, which is influenced by environmental temperature (Nicolson and Thornburg 2007). Consequently, the optimal sugar concentration can change throughout the day and throughout the flowering season. Additionally, the preferences of individual pollinators can shift to higher or lower concentrations, depending on the cost of transportation between flowering plants and the pollinator's water stress (Pivnick and McNeil 1985).

Neither of the two statistical models showed an influence of total AA concentration on plant fitness (Table 3, Fig. 2). This result is surprising as G. conopsea is pollinated by butterflies and moths (Huber et al. 2004), which strongly suggests a potentially important role of nectar AA content on pollinator attraction (Baker and Baker 1986), especially in landscapes with low nitrogen availability, such as calcareous grasslands (Jervis and Boggs 2005). The absence of phenotypic selection on total nectar AA content can have two non-mutually exclusive explanations. First, the effect of nectar AA on the fecundity of adult butterflies is influenced by their nitrogen acquisition during the larval stage, and nectar amino acids only enhance fecundity of adults raised on a nitrogen deprived diet (Jervis and Boggs 2005). The calcareous grasslands of our study area are highly fragmented, dominantly by intensive agricultural land use (Adriaens et al. 2006). Hence pollinators might not be nitrogen deprived if larvae fed on host plants in the nitrogen enriched soils of the surrounding area. Second, it has been experimentally shown that Lepidoptera prefer nectar containing AAs, over nectar lacking AAs (Alm et al. 1990, Mevi- 
Schütz and Erhardt 2003, Cahenzli and Erhardt 2013). To our knowledge, however, no choice experiments have been conducted where butterflies were offered nectars with a different AA content, and it is unknown whether pollinators can distinguish between more subtle differences in AA content.

In a former study in the same study area, nectar AA composition was found to be correlated with fruit set across 12 populations of G. conopsea (Gijbels et al. 2014). However a correlation between fruit set and nectar traits across populations does not indicate a fitness advantage for individual plants as selection acts on among-individual variation within populations. By relating the among-individual variation in trait values to relative female fitness this study is the first to demonstrate phenotypic selection on floral nectar AAs. Our linear regression model showed significant positive selection on PC2 that was positively related to several AAs (Table 3). Two of these AAs, Ala and Ser, also showed significant selection in the structural equation model. Surprisingly, there was a negative selection effect on Ala in the structural equation model whereas Ala concentration positively loaded on PC2, which was under positive selection. These contrasting results are likely a consequence of the statistical approaches used as multiple AAs were loaded on a single component, obscuring the effects of individual AA. Overall, however, our results strengthen the idea that individual nectar AAs have an important role in regulating pollinator behaviour. Positive and negative selection on nectar AA composition can occur as specific AAs attract or deter pollinators, most likely through nectar taste and scent (Petanidou et al. 2006, González-Teuber and Heil 2009, Nepi 2014). Although behavioural responses to nectar AAs were demonstrated for many different pollinator types (Bertazzini et al. 2010, Zhang et al. 2011, Rodríguez-Peña et al. 2013), still very little is known regarding the specific neurological or phago-stimulating effects of different nectar AAs on pollinators in general and Lepidoptera in particular (González-Teuber and Heil 2009, Nepi 2014). Furthermore, we demonstrated phenotypic selection gradients which are likely pollinator-mediated, but may also be influenced by multiple factors such as resource acquisition, antagonist selection or abiotic conditions (Sletvold et al. 2010). We applied different statistical techniques to account for inter-correlations, but these techniques offer no ideal solution for studying phenotypic selection. For instance ordination techniques have the disadvantage of reducing the comparability among studies and obscure selection gradients on individual traits, whereas structural equations require a preselection in variables deemed relevant for the analyses. Still, the two different statistical approaches confirmed the general expectation that nectar composition is subjected to strong selective forces capable of rapidly changing nectar composition (Baker and Baker 1990, Leiss and Klinkhamer 2005, Jacquemyn 
et al. 2012), although selection appears to act more subtle than simply selecting for the best

316 pollinator reward. Phenotypic selection affected individual nectar AAs, and hence AAs appear 317 more important to plant fitness compared to nectar sugars. As there was no selection on total 318 AA concentration, AAs likely do not simply increase pollinator attraction as a nitrogen source, 319 but rather influence pollinator efficiency through pollinator behaviour. We anticipate that future 320 experiments will be able to unravel the details of this process and the importance of AAs in 321 different plant-pollinator relations. Behavioural experiments with different nectar components 322 could address the question how nectar compounds affect different types of pollinators and 323 pollinator mediated selection studies can reveal the consequences of pollinator behaviour on 324 plant reproductive success.

327 PG was funded by the Research Foundation Flanders (FWO). This project was financed 328 through a KULeuven BOF-OT grant. The authors thank Elien Spooren, Timmy Reijnders and 329 Rudy Vergauwen who assisted with field work and HPAEC-PAD analysis and the 330 anonymous reviewers for detailed comments that improved the manuscript. This research 331 benefitted from the KU Leuven Program Financing 'eco and evolutionary dymamics'. 
333

Adriaens, D. et al. 2006. No evidence of a plant extinction debt in highly fragmented calcareous grasslands in Belgium. - Biol. Conserv. 133: 212-224.

Alm, J. et al. 1990. Preference of cabbage white butterflies and honey bees for nectar that contains amino acids. - Oecologia 84: 53-57.

Armbruster, W. S. et al. 2005. Phenotypic selection on dalechampia blossoms: honest signaling affects pollination success. - Ecology 86: 3323-3333.

Baker, H. G. and Baker, I. 1983. Floral nectar sugar constituents in relation to pollinator type. In Jones C. E. and Little R. J. Hand-book of experimental pollination biology, Van Nostrand Reinhold New York, pp. 117-41.Baker, H. G. and Baker, I. 1986. The occurrence and significance of amino acids in floral nectar. - Plant Syst. Evol. 151: 175-186.

Baker, H. G. and Baker, I. 1990. The predictive value of nectar chemistry to the recognition of pollinator types. - Isr. J. Bot. 39: 157-166.

Bertazzini, M. et al. 2010. Amino acid content and nectar choice by forager honeybees (Apis mellifera L.). - Amino Acids 39: 315-318.

Bloch, D. and Erhardt, A. 2008. Selection toward shorter flowers by butterflies whose probosces are shorter than floral tubes. - Ecology 89: 2453-2460.

Brandenburg, A. et al. 2009. The sweetest thing: advances in nectar research. - Curr. Opin. Plant Biol. 12: 486-490.

Cahenzli, F. and Erhardt, A. 2013. Nectar amino acids enhance reproduction in male butterflies. - Oecologia 171: 197-205.

Campbell, D. R. et al. 1996. Mechanisms of Hummingbird-Mediated Selection for Flower width in Ipomopsis aggregata. - Ecology 77: 1463.

Farkas, A. et al. 2012. Variation in nectar volume and sugar concentration of Allium ursinum L. ssp. ucrainicum in three habitats. - ScientificWorldJournal 2012: 138579.

Fenster, C. B. et al. 2004. Pollination syndromes and floral specialization. - Annu. Rev. Ecol. Evol. Syst. 35: 375-403.

Gijbels, P. et al. 2014. Landscape scale variation in nectar amino acid and sugar composition in a Lepidoptera pollinated orchid species and its relation with fruit set. - J. Ecol. 102: $136-144$.

González-Teuber, M. and Heil, M. 2009. Nectar chemistry is tailored for both attraction of mutualists and protection from exploiters. - Plant Signal. Behav. 4: 809-813. 
Grace, J. B. et al. 2007. Does species diversity limit productivity in natural grassland communities? - Ecol. Lett. 10: 680-689.

Gustafsson, S. 2000. Patterns of genetic variation in Gymnadenia conopsea, the fragrant orchid. - Mol. Ecol. 9: 1863-1872.

Harder, L. D. and Johnson, S. D. 2009. Darwin's beautiful contrivances: evolutionary and functional evidence for floral adaptation. - New Phytol. 183: 530-545.

Heil, M. 2011. Nectar: generation, regulation and ecological functions. - Trends Plant Sci. 16: 191-200.

Herrera, C. M. et al. 2006. Extreme intraplant variation in nectar sugar composition in an insect-pollinated perennial herb. - Am. J. Bot. 93: 575-581.

Huber, F. K. et al. 2004. Floral scent emission and pollinator attraction in two species of Gymnadenia (Orchidaceae). - Oecologia 142: 564-575.

Jacquemyn, H. et al. 2012. Evolutionary changes in plant reproductive traits following habitat fragmentation and their consequences for population fitness: Habitat fragmentation and plant trait evolution. - J. Ecol. 100: 76-87.

Jervis, M. A. and Boggs, C. L. 2005. Linking nectar amino acids to fitness in female butterflies. - Trends Ecol. Evol. 20: 585-587.

Johnson, S. D. and Steiner, K. E. 1997. Long-tongued fly pollination and evolution of floral spur length in the Disa draconis complex (Orchidaceae). - Evolution 51: 45.

Knight, T. M. et al. 2005. Pollen limitation of plant reproduction: Pattern and Process. - Annu. Rev. Ecol. Evol. Syst. 36: 467-497.

Komarov, V. 1986. Flora of the USSR.

Krömer, T. et al. 2008. Nectar sugar composition and concentration in relation to pollination syndromes in Bromeliaceae. - Plant Biol. 10: 502-511.

Lande, R. and Arnold, S. J. 1983. The measurement of selection on correlated characters. Evolution 37: 1210.

Leiss, K. A. and Klinkhamer, P. G. L. 2005. Spatial distribution of nectar production in a natural Echium vulgare population: Implications for pollinator behaviour. - Basic Appl. Ecol. 6: 317-324.

Meekers, T. et al. 2012. Biological flora of the British Isles: Gymnadenia conopsea s.l. - J. Ecol. 100: 1269-1288.

Mevi-Schütz, J. and Erhardt, A. 2003. Effects of nectar amino acids on fecundity of the wall brown butterfly (Lasiommata megera L.). - Basic Appl. Ecol. 4: 413-421. 
Mitchell, R. J. et al. 2004. The influence of Mimulus ringens floral display size on pollinator visitation patterns. - Funct. Ecol. 18: 116-124.

Nepi, M. 2014. Beyond nectar sweetness: the hidden ecological role of non-protein amino acids in nectar. - J. Ecol. 102: 108-115.

Nicolson, S. W. and Thornburg, R. W. 2007. Nectar chemistry. - In: Nicolson, S. W. et al. (eds), Nectaries and Nectar. Springer Netherlands, pp. 215-264.

Nocentini, D. et al. 2013. Intrapopulation heterogeneity in floral nectar attributes and foraging insects of an ecotonal Mediterranean species. - Plant Ecol. 214: 799-809.

Petanidou, T. 2005. Sugars in Mediterranean floral nectars: an ecological and evolutionary approach. - J. Chem. Ecol. 31: 1065-1088.

Petanidou, T. et al. 2006. What shapes amino acid and sugar composition in Mediterranean floral nectars? - Oikos 115: 155-169.

Pivnick, K. A. and McNeil, J. N. 1985. Effects of nectar concentration on butterfly feeding: measured feeding rates for Thymelicus lineola (Lepidoptera: Hesperiidae) and a general feeding model for adult Lepidoptera. - Oecologia 04: 226-237.

Quinn, G. P. 2002. Experimental design and data analysis for biologists. - Cambridge University Press.

Rodríguez-Peña, N. et al. 2013. Nitrogen and amino acids in nectar modify food selection of nectarivorous bats. - J. Anim. Ecol. 82: 1106-1115.

Rusterholz, H.-P. and Erhardt, A. 1997. Preferences for nectar sugars in the peacock butterfly, Inachis io. - Ecol. Ent. 22, 220-224.

Scheiner et al. 2000. Using path analysis to measure natural selection. - J. Evol. Biol. 13: 423-433.

Sinclair, S. J. 2012. Low-nutrient soils, pollination and plant diversity. - J. Veg. Sci. 23: 585589.

Sletvold, N. and Ågren, J. 2010. Pollinator-mediated selection on floral display and spur length in the orchid Gymnadenia conopsea. - Int. J. Plant Sci. 171: 999-1009.

Sletvold, N. et al. 2010. Pollinator-mediated selection on floral display, spur length and flowering phenology in the deceptive orchid Dactylorhiza lapponica. - New Phytol. 188: 385-392.

Sletvold, N. et al. 2012. Separating selection by diurnal and nocturnal pollinators on floral display and spur length in Gymnadenia conopsea. - Ecology 93: 1880-1891.

Sletvold, N. et al. 2013. Vegetation context influences the strength and targets of pollinatormediated selection in a deceptive orchid. - Ecology 94: 1236-1242. 
432 Thompson, J. N. 2005. Coevolution: the geographic mosaic of coevolutionary arms races. Curr. Biol. 15: R992-R994.

434 Wolff, D. 2006. Nectar sugar composition and volumes of 47 species of gentianales from a southern ecuadorian montane forest. - Ann. Bot. 97: 767-777.

436 Zhang, Y.-F. et al. 2011. Tarsal taste neurons of Helicoverpa assulta (Guenée) respond to sugars and amino acids, suggesting a role in feeding and oviposition. - J. Insect Physiol. 57: 1332-1340.

439 
441 Figure 1: Standardized linear selection gradients of the two significant variables inflorescence

442 height and PC2 in 132 individuals of G. conopsea. Selection gradients are illustrated with 443 added-variable plots.
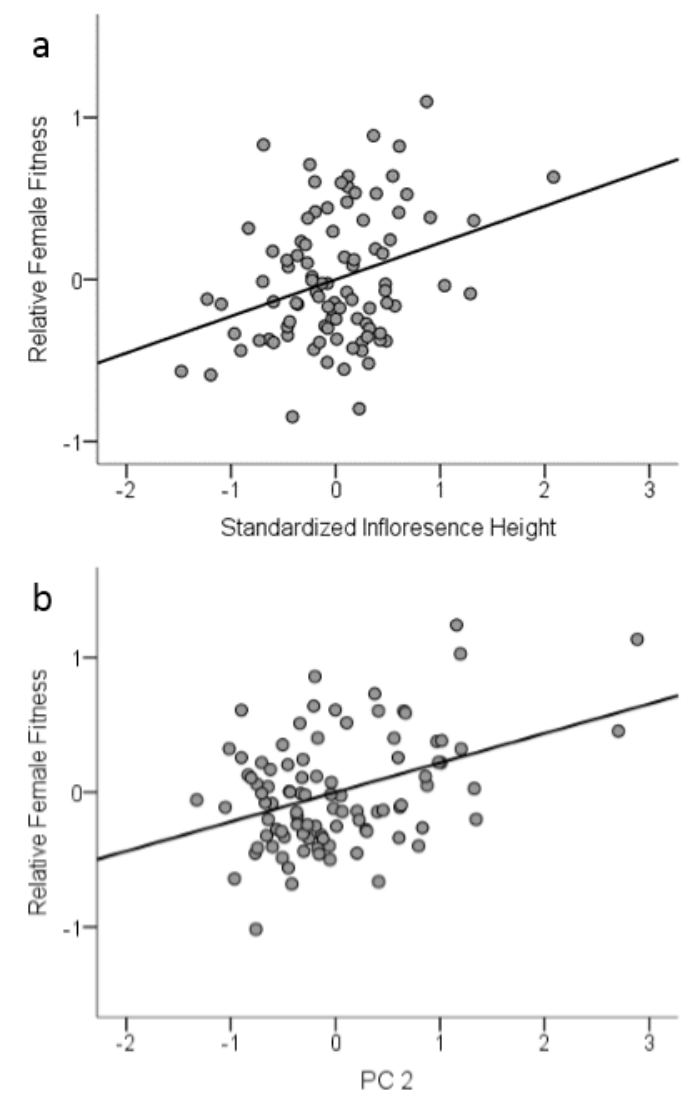
447 Figure 2: Path analyses of the best structural equation model (model 3 of Table 4). Excluded 448 variables are indicated with dotted lines, black arrows denote positive effects, red arrows denote 449 negative effects and arrow widths are proportional to the standardized path coefficients, 450 significance is indicated (*: $\mathrm{P}<0.05$, ***: $\mathrm{P}<0.01)$. Variables in the blue area are all 451 significantly intercorrelated (AA: total amino acid concentration $(\mu \mathrm{M})$, Ala: alanine, Asn: 452 Asparagine, Gln: glutamine, His: histidine, Ile: isoleucine, Lys: lysine, Ser: serine, Thr: 453 threonine).

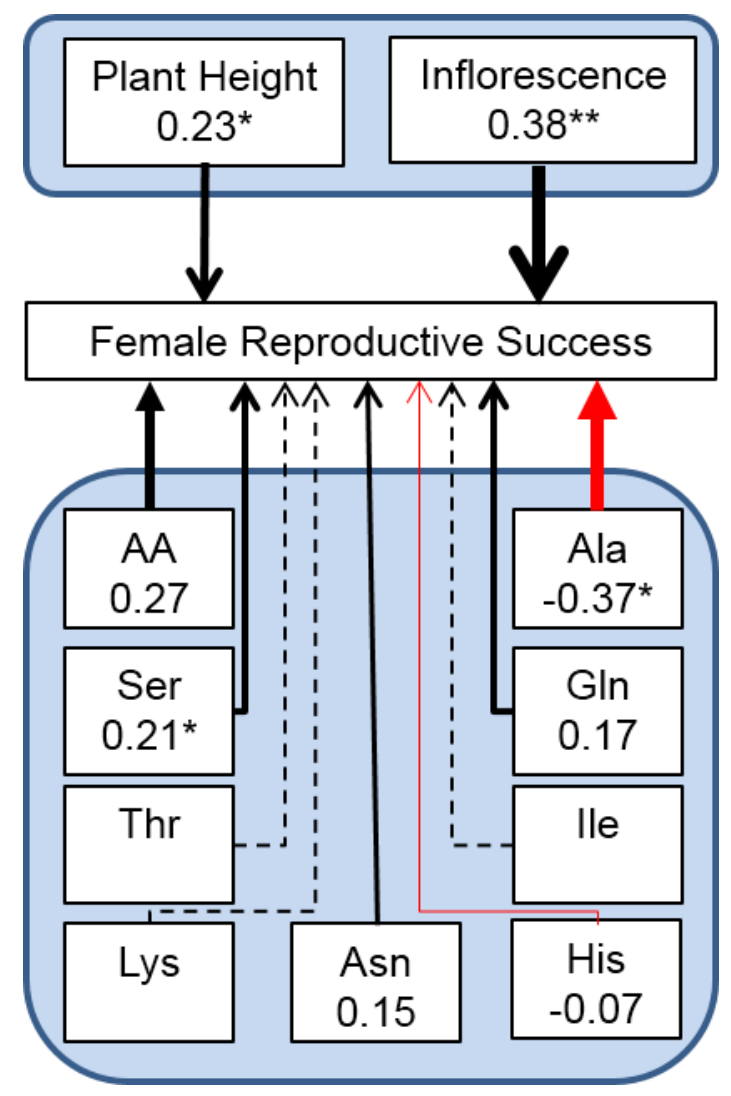


457 Table 1: Population characteristics and average \pm S.D. of phenotypic traits in three populations 458 of $G$. conopsea. F-value are associated with the difference between populations obtained by a 459 one-way ANOVA, significance is indicated.

\begin{tabular}{|c|c|c|c|c|}
\hline & Coupu Tiene & Les Hurée & Gay & $\mathrm{F}$ \\
\hline Location & $\begin{array}{l}50^{\circ} 07^{\prime} 25.55^{\prime \prime} \mathrm{N} \\
04^{\circ} 41^{\prime} 53.03 ” \mathrm{E}\end{array}$ & $\begin{array}{l}50^{\circ} 06^{\prime} 42.84 ” \mathrm{~N} \\
04^{\circ} 37^{\prime} 21.96^{\prime} \mathrm{E}\end{array}$ & $\begin{array}{l}50^{\circ} 05^{\prime} 41.74 " \mathrm{~N} \\
04^{\circ} 40^{\prime} 16.84^{\prime \prime} \mathrm{E}\end{array}$ & \\
\hline Flowering individuals & 541 & 279 & 56 & \\
\hline Sampled individuals & 50 & 48 & 34 & \\
\hline Fertilized fruits & $29.6 \pm 15.0$ & $32.1 \pm 12.3$ & $29.1 \pm 9.8$ & 0.61 \\
\hline Fruit weight (mg) & $10.2 \pm 3.8$ & $14.5 \pm 5.2$ & $9.9 \pm 2.2$ & $16.30 * * *$ \\
\hline Female Fitness & $0.30 \pm 0.18$ & $0.49 \pm 0.26$ & $0.26 \pm 0.14$ & $12.06 * * *$ \\
\hline Spur (mm) & $16.69 \pm 1.85$ & $17.07 \pm 1.40$ & $16.80 \pm 1.6$ & 0.70 \\
\hline Height (cm) & $34 \pm 7$ & $41 \pm 9$ & $40 \pm 7.6$ & $10.50 * * *$ \\
\hline Inflorescence $(\mathrm{cm})$ & $10.3 \pm 3.0$ & $10.1 \pm 3.0$ & $10.8 \pm 3.2$ & 0.60 \\
\hline Sugar (mM) & $690 \pm 194$ & $347 \pm 109$ & $697 \pm 154$ & $73.39 * * *$ \\
\hline Sucrose & $550 \pm 173$ & $265 \pm 88$ & $565 \pm 82$ & $54.72 * * *$ \\
\hline $\mathrm{AA}(\mu \mathrm{M})$ & $7609 \pm 4794$ & $6722 \pm 4182$ & $9035 \pm 4501$ & 2.45 \\
\hline Alanine & $996 \pm 552$ & $900 \pm 643$ & $1461 \pm 758$ & $7.61 * *$ \\
\hline Arginine & $505 \pm 704$ & $241 \pm 153$ & $452 \pm 414$ & $3.69 *$ \\
\hline Asparagine & $181 \pm 207$ & $341 \pm 325$ & $217 \pm 146$ & $5.07 * *$ \\
\hline Aspartic acid & $417 \pm 574$ & $417 \pm 390$ & $440 \pm 544$ & 0.025 \\
\hline Glutamic acid & $836 \pm 1334$ & $468 \pm 346$ & $406 \pm 341$ & 3.05 \\
\hline Glutamine & $199 \pm 220$ & $424 \pm 596$ & $257 \pm 181$ & $3.82 *$ \\
\hline Glycine & $3015 \pm 2135$ & $2655 \pm 1904$ & $4487 \pm 2772$ & $6.63 * *$ \\
\hline Histidine & $103 \pm 110$ & $89 \pm 94.7$ & $240 \pm 268$ & 9.39 \\
\hline Isoleucine & $38 \pm 63$ & $36 \pm 37$ & $24 \pm 31$ & 0.82 \\
\hline Leucine & $78 \pm 106$ & $48 \pm 40$ & $57 \pm 52$ & 1.93 \\
\hline Lysine & $167 \pm 129$ & $166 \pm 125$ & $117 \pm 72$ & 2.09 \\
\hline Ornithine & $57 \pm 55.6$ & $42 \pm 54$ & $32 \pm 34$ & 2.12 \\
\hline Phenylalanine & $101 \pm 163$ & $40 \pm 35$ & $61 \pm 66$ & $3.77 *$ \\
\hline Serine & $450 \pm 342$ & $597 \pm 609$ & $485 \pm 405$ & 1.17 \\
\hline Threonine & $85 \pm 70$ & $79 \pm 65$ & $65 \pm 48$ & 0.91 \\
\hline
\end{tabular}




\begin{tabular}{lclll} 
Tyrosine & $90 \pm 132$ & $47 \pm 35$ & $62 \pm 69$ & 2.69 \\
Valine & $270 \pm 217$ & $110 \pm 101$ & $157 \pm 139$ & $11.62 * * *$ \\
\hline$*: \mathrm{P}<0.05, * *: \mathrm{P}<0.01, * * *: \mathrm{P}<0.001$ & &
\end{tabular}

460

461 
462 Table 2: Principal component analyses with varimax rotation of the AA concentrations in 132

463 individuals across three populations of G. conopsea. The component matrix shows the relative 464 values of the AAs, the eigenvalues and the ratio of total variance of each principal component.

465 Bold scripts show the principal component that matches the different variables best.

466

\begin{tabular}{lccc}
\hline Variables & PC1 & PC2 & PC3 \\
\hline AA & 0.57 & 0.47 & $\mathbf{0 . 6 4}$ \\
Alanine & 0.11 & $\mathbf{0 . 7 4}$ & 0.56 \\
Arginine & $\mathbf{0 . 8 9}$ & 0.24 & 0.06 \\
Asparagine & 0.13 & $\mathbf{0 . 8}$ & 0.01 \\
Aspartic acid & $\mathbf{0 . 7 3}$ & 0.13 & 0.19 \\
Glutamic acid & $\mathbf{0 . 8 6}$ & 0.16 & 0.13 \\
Glutamine & 0.16 & $\mathbf{0 . 8 5}$ & 0.01 \\
Glycine & 0.1 & 0.1 & $\mathbf{0 . 9 6}$ \\
Histidine & $\mathbf{0 . 8 3}$ & 0.35 & 0.11 \\
Isoleucine & 0.51 & $\mathbf{0 . 6 6}$ & 0.12 \\
Leucine & 0.52 & $\mathbf{0 . 6 3}$ & 0.25 \\
Lysine & 0.51 & $\mathbf{0 . 6 7}$ & 0.32 \\
Ornithine & $\mathbf{0 . 8 5}$ & 0.08 & 0.17 \\
Phenylalanine & $\mathbf{0 . 8 9}$ & 0.3 & 0.01 \\
Serine & 0.16 & $\mathbf{0 . 8 9}$ & 0.09 \\
Threonine & 0.28 & $\mathbf{0 . 7 7}$ & 0.36 \\
Tyrosine & $\mathbf{0 . 8 6}$ & 0.3 & 0.02 \\
Valine & 0.55 & $\mathbf{0 . 6 7}$ & 0.07 \\
Eigenvalue & 10.5 & 2.5 & 1.3 \\
\% of Variance & 58.3 & 14.0 & 7.3 \\
\hline & & & \\
\hline
\end{tabular}


468 Table 3: Multivariate linear selection coefficients on female reproductive success in 132 469 individuals across three populations of G. conopsea.

470

\begin{tabular}{lcc}
\hline Parameter & Linear selection gradient $(\beta) \pm$ S.E. & $\mathrm{F}$ \\
\hline Plant height & $0.12 \pm 0.07$ & 2.58 \\
Inflorescence & $0.23 \pm 0.07^{* *}$ & 9.51 \\
Spur length & $-0.04 \pm 0.05$ & 0.58 \\
Sugar & $0.06 \pm 0.05$ & 1.80 \\
Sucrose & $-0.06 \pm 0.05$ & 1.51 \\
PC1 & $-0.01 \pm 0.07$ & 0.01 \\
PC2 & $0.22 \pm 0.06^{* *}$ & 15.48 \\
PC3 & $-0.01 \pm 0.06$ & 0.01 \\
\hline$* *:$ P $<0.01 ;$ & &
\end{tabular}

471

472 
473 Table 4: Summary of model selection in structural equation modelling. Lowest Chisq/Df and 474 highest GFI and Model P-value indicate best model fit.

475

\begin{tabular}{lcccr}
\hline Model & Chisq/Df & GFI & $\begin{array}{r}\text { Model } \\
\text { P-Value }\end{array}$ & $\begin{array}{r}\text { Least significant variable } \\
\text { (P-value) }\end{array}$ \\
\hline Saturated & 2.11 & 980 & 0.01 & Threonine (0.79) \\
Model 1 & 2.01 & 980 & 0.01 & Lysine (0.47) \\
Model 2 & 2.28 & 973 & 0.01 & Isoleucine (0.48) \\
Model 3 & 1.39 & 992 & 0.16 & Histidine $(0.49)$ \\
Model 4 & 1.62 & 988 & 0.09 & Total AA $(0.21)$ \\
Model 5 & 1.96 & 979 & 0.05 & Alanine $(0.15)$ \\
Model 6 & 2.37 & 973 & 0.03 & Serine $(0.12)$ \\
\hline
\end{tabular}

